\title{
STANDARD COMPLEX FOR QUANTUM LIE ALGEBRAS
}

\author{
C. BURDIK ${ }^{a}$, A.P. ISAEV ${ }^{b}$ and O. OGIEVETSKY ${ }^{c}$ \\ ${ }^{a}$ Department of Mathematics, Faculty of Nuclear Sciences and Physical \\ Engineering, Trojanova 13, 12000 Prague 2, Czech Republic \\ E-mail: burdik@dec1.fjfi.cvut.cz \\ ${ }^{b}$ Bogoliubov Theoretical Laboratory, Joint Institute for Nuclear Research, \\ Dubna, Moscow region 141980, Russia \\ E-mail: isaevap@thsun1.jinr.ru \\ ${ }^{c}$ Center of Theoretical Physics, Luminy, 13288 Marseille, France \\ and P. N. Lebedev Physical Institute, Theoretical Department, Leninsky \\ pr. 53, 117924 Moscow, Russia \\ E-mail: oleg@cpt.univ-mrs.fr
}

\begin{abstract}
For a quantum Lie algebra $\Gamma$, let $\Gamma^{\wedge}$ be its exterior extension (the algebra $\Gamma^{\wedge}$ is canonically defined). We introduce a differential on the exterior extension algebra $\Gamma^{\wedge}$ which provides the structure of a complex on $\Gamma^{\wedge}$. In the situation when $\Gamma$ is a usual Lie algebra this complex coincides with the "standard complex". The differential is realized as a commutator with a (BRST) operator $Q$ in a larger algebra $\Gamma^{\wedge}[\Omega]$, with extra generators canonically conjugated to the exterior generators of $\Gamma^{\wedge}$. A recurrent relation which defines uniquely the operator $Q$ is given.
\end{abstract}


1. A quantum Lie algebra [1], [2], [3], 四] is defined by two tensors $C_{i j}^{k}$ and $\sigma_{i j}^{m k}$ (indices belong to some set $\mathcal{N}$, say, $\mathcal{N}=\{1, \ldots, N\}$ ). By definition, the matrix $\sigma_{i j}^{m k}$ has an eigenvalue 1 ; one demands that $\left(P_{(1)}\right)_{i j}^{m k} C_{m k}^{n}=0$, where $P_{(1)}$ is a projector on the eigenspace of $\sigma$ corresponding to the eigenvalue 1 .

By definition, a quantum Lie algebra $\Gamma$ is generated by elements $\chi_{i}, i=$ $1, \ldots, N$, subjected to relations

$$
\chi_{i} \chi_{j}-\sigma_{i j}^{m k} \chi_{m} \chi_{k}=C_{i j}^{k} \chi_{k} .
$$

Here the structure constants $C_{i j}^{k}$ obey

$$
\begin{gathered}
C_{n i}^{p} C_{p j}^{l}=\sigma_{i j}^{m k} C_{n m}^{p} C_{p k}^{l}+C_{i j}^{p} C_{n p}^{l} \Leftrightarrow \\
\Leftrightarrow C_{\mid 12>}^{<1 \mid} C_{\mid 13>}^{<4 \mid}=\sigma_{23} C_{\mid 12>}^{<1 \mid} C_{\mid 13>}^{<4 \mid}+C_{\mid 23>}^{<3 \mid} C_{\mid 13>}^{<4 \mid}, \\
C_{n i}^{k} \sigma_{k q}^{p m}=\sigma_{i q}^{s j} \sigma_{n s}^{p k} C_{k j}^{m} \Leftrightarrow C_{\mid 12>}^{<1 \mid} \sigma_{13}=\sigma_{23} \sigma_{12} C_{\mid 23>}^{<3 \mid}, \\
\left(\sigma_{i m}^{p j} C_{q p}^{n}+\delta_{q}^{n} C_{i m}^{j}\right) \sigma_{n j}^{k s}=\sigma_{q i}^{j n}\left(\sigma_{n m}^{p s} C_{j p}^{k}+\delta_{j}^{k} C_{n m}^{s}\right) \Leftrightarrow \\
\Leftrightarrow \quad\left(\sigma_{23} C_{\mid 12>}^{<1 \mid}+C_{\mid 23>}^{<3 \mid}\right) \sigma_{13}=\sigma_{12}\left(\sigma_{23} C_{\mid 12>}^{<1 \mid}+C_{\mid 23>}^{<3 \mid}\right) .
\end{gathered}
$$

The matrix $\sigma_{i j}^{m k}$ satisfies the Yang-Baxter equation

$$
\sigma_{i_{1} i_{2}}^{j_{1} j_{2}} \sigma_{j_{2} i_{3}}^{n_{2} k_{3}} \sigma_{j_{1} n_{2}}^{k_{1} k_{2}}=\sigma_{i_{2} i_{3}}^{j_{2} j_{3}} \sigma_{i_{1} j_{2}}^{k_{1} n_{2}} \sigma_{n_{2} j_{3}}^{k_{2} k_{3}} \Leftrightarrow \sigma_{12} \sigma_{23} \sigma_{12}=\sigma_{23} \sigma_{12} \sigma_{23} .
$$

In the right hand side of (3)-(可) we use FRT matrix notations [5]; $\{1,2,3, \ldots\}$ are the numbers of vector spaces, e.g., $f_{1}:=f_{j_{1}}^{i_{1}}$ is a matrix which acts in the first vector space. Additionally, we use incoming and outcoming indices, e.g., $\Omega^{<1 \mid}:=\Omega^{i_{1}}$ and $\gamma_{\mid 1>}:=\gamma_{j_{1}}$ denote a covector with one outcoming index and a vector with one incoming index respectively. Thus, in this notation, the matrix $f_{1}$ can be written as $f_{1}=f_{\mid 1>}^{<1 \mid}$.

Remark. Quantum Lie algebras defined by equations (11)-(5) generalize the usual Lie (super-)algebras. Indeed in the non-deformed case, when

$$
\sigma_{i j}^{m k}=(-1)^{(m)(k)} \delta_{j}^{m} \delta_{i}^{k}
$$

is a super-permutation matrix (here $\sigma^{2}=1$ and (5) is fulfilled; $(m)=0,1$ is the parity of a generator $\chi_{m}$ ), equations (11) and (2) coincide with the defining 
relations and the Jacobi identities for Lie (super)-algebras. Equation (3) is then equivalent to the $Z_{2}$-homogeneity condition $C_{j k}^{i}=0$ for $(i) \neq(j)+(k)$. Equation (4) follows from (3).

2. The exterior extension $\Gamma^{\wedge}$ of the quantum algebra $\Gamma$ (1) is obtained by adding new generators $\gamma_{i}, i=1, \ldots, N$. The generators $\gamma_{i}$ form a "generalized" wedge algebra. The definition of the wedge product of the elements $\gamma_{i}$ is

$$
\gamma_{\mid 1>} \wedge \gamma_{\mid 2>} \ldots \wedge \gamma_{\mid n>}=A_{1 \rightarrow n} \gamma_{\mid 1>} \otimes \gamma_{\mid 2>} \ldots \otimes \gamma_{\mid n>}
$$

Here the matrix operator $A_{1 \rightarrow n}$ is an analog of the antisymmetrizer of $n$ spaces. This operator can be defined inductively (see e.g. [6])

$$
A_{1 \rightarrow n}=\left(\mathbf{1}+\sum_{k=1}^{n-1}(-1)^{n-k} \sigma_{k \rightarrow n}\right) A_{1 \rightarrow n-1}
$$

where, for $n>k$,

$$
\sigma_{k \rightarrow n}:=\sigma_{k k+1} \sigma_{k+1 k+2} \ldots \sigma_{n-1 n} .
$$

Using the Yang-Baxter equation (5) one can rewrite (7) in the following three equivalent forms

$$
\begin{gathered}
A_{1 \rightarrow n}=A_{1 \rightarrow n-1}\left(\mathbf{1}+\sum_{k=1}^{n-1}(-1)^{k} \sigma_{k+1 \leftarrow 1}\right) \\
=\left(\mathbf{1}+\sum_{k=1}^{n-1}(-1)^{k} \sigma_{k+1 \leftarrow 1}\right) A_{2 \rightarrow n}=A_{2 \rightarrow n}\left(\mathbf{1}+\sum_{k=1}^{n-1}(-1)^{n-k} \sigma_{k \rightarrow n}\right)
\end{gathered}
$$

where

$$
\sigma_{n \leftarrow k}:=\sigma_{n-1 n} \ldots \sigma_{k+1 k+2} \sigma_{k k+1}
$$

for $n>k$.

If the sequence of operators $A_{1 \rightarrow n}$ terminates at the step $n=h+1$ $\left(A_{1 \rightarrow h} \neq 0\right.$ and $A_{1 \rightarrow n}=0$ for $\left.n>h\right)$ then the number $h$ is called the height of the operator $\sigma$.

The cross-commutation relations between the generators $\gamma_{i}$ and $\chi_{j}$ are:

$$
\gamma_{\mid 1>} \chi_{\mid 2>}=\left(\sigma_{12} \chi_{\mid 1>}+C_{\mid 12>}^{<2 \mid}\right) \gamma_{\mid 2>}
$$

The algebra $\Gamma^{\wedge}$ is graded by the degree in the generators of $\gamma_{i}$. 
3. We further introduce a set of generators $\left\{\Omega^{i}\right\}, i=1, \ldots, N$, canonically conjugated to the generators $\gamma_{i}$. The generators $\Omega^{i}$ form a "wedge" algebra as well, with the wedge product defined by

$$
\Omega^{<r \mid} \wedge \Omega^{<r-1 \mid} \wedge \ldots \wedge \Omega^{<1 \mid}=\Omega^{<r \mid} \otimes \Omega^{<r-1 \mid} \otimes \ldots \otimes \Omega^{<1 \mid} A_{1 \rightarrow r} .
$$

Here operators $A_{1 \rightarrow n}$ are the same as in (7).

The commutation relations between $\Omega^{i}$ and $\gamma_{j}$ are

$$
\gamma_{j} \Omega^{i}=-\Omega^{p}\left(\sigma^{-1}\right)_{p j}^{s i} \gamma_{s}+\delta_{j}^{i} \Rightarrow \gamma_{\mid 2>} \Omega^{<2 \mid}=-\Omega^{<1 \mid} \sigma_{12}^{-1} \gamma_{\mid 1>}+I_{2} .
$$

Finally the commutation relations between $\Omega^{i}$ and $\chi_{j}$ are

$$
\chi_{\mid 2>} \Omega^{<2 \mid}=\Omega^{<1 \mid}\left(\sigma_{12} \chi_{\mid 1>}+C_{\mid 12>}^{<2 \mid}\right) .
$$

We denote the algebra generated by $\left\{\chi_{i}\right\},\left\{\gamma_{j}\right\}$ and $\left\{\Omega^{k}\right\}$ by $\Gamma^{\wedge}[\Omega]$. The algebra $\Gamma^{\wedge}[\Omega]$ is graded by the rule: $\operatorname{deg}\left(\gamma_{\mathrm{i}}\right)=1$ and $\operatorname{deg}\left(\Omega^{\mathrm{i}}\right)=-1$.

We shall need the following set of consequences of the equation (10):

$$
\begin{aligned}
\gamma_{\mid 1>} \wedge \ldots \wedge \gamma_{\mid r>} \Omega^{<r \mid}=(-1)^{r} \Omega^{<0 \mid} \sigma_{r \leftarrow 0}^{-1} \gamma_{\mid 0>} \wedge \ldots \wedge \gamma_{\mid r-1>} \\
+\left(\sum_{k=1}^{r}(-1)^{r-k} \sigma_{r \leftarrow k}^{-1}\right) \gamma_{\mid 1>} \wedge \ldots \wedge \gamma_{\mid r-1>},
\end{aligned}
$$

where $\sigma_{r \leftarrow k}^{-1}:=\sigma_{k k+1}^{-1} \ldots \sigma_{r-1 r}^{-1}$ and $\sigma_{r \leftarrow r}^{-1}:=\mathbf{1}$.

4. The main result of the present paper is a recursive formula for the BRST operator $Q$ which satisfies $Q^{2}=0$.

Such an operator endows the algebra $\Gamma^{\wedge}$ with the structure of the differential (chain) complex. To construct the differential (starting with the operator $Q$ ) one needs first to define the action of the algebra $\Gamma^{\wedge}[\Omega]$ on the algebra $\Gamma^{\wedge}$. The elements $\chi_{i}$ and $\gamma_{j}$ act on $\Gamma^{\wedge}$ by the left multiplication. To define the action of generators $\Omega^{i}$ on $\Gamma^{\wedge}$ it suffices (due to relations (10) and (11)) to know $\Omega^{i}(1)$, where 1 is the unit element of the algebra $\Gamma^{\wedge}$. We set $\Omega^{i}(1)=0$. The definition of the differential $d$ is given by its action on an element $\phi$ of the algebra $\Gamma^{\wedge}$,

$$
d \phi=[Q, \phi]_{ \pm}(1)
$$

where $[,]_{ \pm}$is the graded commutator. 
Now we are ready to formulate the main Proposition.

Proposition. The BRST operator $Q$ for the quantum algebra (1) has the following form

$$
Q=\Omega^{i} \chi_{i}+\sum_{r=1}^{h-1} Q_{(r)},
$$

where $h$ is the height of the operator $A_{1 \rightarrow n}$ (1).

Here the operators $Q_{(r)}$ are given by

$$
Q_{(r)}=\Omega^{<r+1 \mid} \Omega^{<r \mid} \ldots \Omega^{<1 \mid} X_{\mid 1 \ldots r+1>}^{<\tilde{1} \ldots \tilde{r} \mid} \gamma_{\mid \tilde{1}>} \cdots \gamma_{\mid \tilde{r}>}
$$

(the wedge product is implied); $X_{\mid 1 \ldots r+1>}^{<1 \ldots r \mid}$ are tensors which satisfy the following recurrent relations

$$
A_{1 \rightarrow r+1} X_{\mid 1 \ldots r+1>}^{<1 \ldots r \mid} A_{1 \rightarrow r}=A_{1 \rightarrow r+1}\left((-1)^{r} \sigma_{r+1 \leftarrow 1}-1\right) X_{\mid 2 \ldots r+1>}^{<2 \ldots r \mid} A_{2 \rightarrow r}
$$

with the initial condition $A_{12} X_{\mid 12>}^{<0 \mid}=-C_{\mid 12>}^{<0 \mid}$.

Proof. We have to verify the identity

$$
Q^{2}=\left(\Omega^{<2 \mid} \chi_{\mid 2>}\right)^{2}+\left[\Omega^{<2 \mid} \chi_{\mid 2>}, \sum_{r=1}^{h-1} Q_{(r)}\right]_{+}+\left(\sum_{r=1}^{h-1} Q_{(r)}\right)^{2}=0 .
$$

Because of the lack of space we shall check a part of this identity which includes the linear in $\chi$ terms only.

First of all we find (see (11))

$$
\begin{gathered}
\left(\Omega^{<2 \mid} \chi_{\mid 2>}\right)^{2}=\Omega^{<2 \mid}\left(\Omega^{<1 \mid}\left(\sigma_{12} \chi_{\mid 1>}+C_{\mid 12>}^{<2 \mid}\right)\right) \chi_{\mid 2>} \\
=\Omega^{<2 \mid} \otimes \Omega^{<1 \mid} \sigma_{12} C_{\mid 12>}^{<2 \mid} \chi_{\mid 2>}+\Omega^{<2 \mid} \otimes \Omega^{<1 \mid}(1-\sigma)_{12} C_{\mid 12>}^{<2 \mid} \chi_{\mid 2>} \\
=\Omega^{<2 \mid} \otimes \Omega^{<1 \mid} C_{\mid 12>}^{<2 \mid} \chi_{\mid 2>} .
\end{gathered}
$$

Consider then the anticommutator $\left[\Omega^{<2 \mid} \chi_{\mid 2>}, Q_{(r)}\right]_{+}$in which we commute all $\chi_{i}$ to the right and extract only the terms which are linear in the generators $\chi_{i}$ :

$$
\left[Q_{(r)}, \Omega^{<r \mid} \chi_{\mid r>}\right]_{+}=\Omega^{<r+1 \mid} \chi_{\mid r+1>} Q_{(r)}+Q_{(r)} \Omega^{<r \mid} \chi_{\mid r>}
$$




$$
\begin{gathered}
=\Omega^{<r+1 \mid} \ldots \Omega^{<0 \mid}\left(\sigma_{r+1 \leftarrow 0}+(-1)^{r} \mathbf{1}\right) X_{\mid 1 \ldots r+1>}^{<1 \ldots r \mid} \sigma_{r \leftarrow 0}^{-1} \gamma_{\mid 0>} \ldots \gamma_{\mid r-1>} \chi_{\mid r>} \\
+\Omega^{<r+1 \mid} \ldots \Omega^{<1 \mid} X_{\mid 1 \ldots r+1>}^{<1 \ldots r \mid}\left(\sum_{k=1}^{r}(-1)^{r-k} \sigma_{r \leftarrow k}^{-1}\right) \gamma_{\mid 1>} \ldots \gamma_{\mid r-1>} \chi_{\mid r>}+\ldots
\end{gathered}
$$

(dots denote the terms independent of $\chi_{i}$ ). Here eqs. (11), (8) and (12) have been used.

Equations (19) and (18) give the whole contribution to the $\chi$-linear terms in $Q^{2}$ since $\left(\sum_{r=1}^{h-1} Q_{(r)}\right)^{2}$ is independent of $\chi_{i}$.

The substitution of (18) and (19) produces the initial data $A_{12} X_{\mid 12>}^{<0 \mid}=$ $-C_{\mid 12>}^{<0 \mid}$ and recurrent relations

$$
\begin{gathered}
A_{1 \rightarrow r+1} X_{\mid 1 \ldots r+1>}^{<1 \ldots r \mid}\left(\sum_{k=1}^{r}(-1)^{r-k} \sigma_{r \leftarrow k}^{-1}\right) A_{1 \rightarrow r-1} \\
=-A_{1 \rightarrow r+1}\left(\sigma_{r+1 \leftarrow 1}+(-1)^{r-1} \mathbf{1}\right) X_{\mid 2 \ldots r+1>}^{<2 \ldots r \mid} \sigma_{r \leftarrow 1}^{-1} A_{1 \rightarrow r-1},
\end{gathered}
$$

where the matrix operator $A_{1 \rightarrow r}$ is defined in (7). These relations express coefficients $X_{\mid 1 \ldots r+1>}^{<1 \ldots r \mid}$ via $X_{\mid 1 \ldots r>}^{<1 \ldots r-1 \mid}$.

Using an identity $\sigma_{r \leftarrow 1}^{-1} A_{1 \rightarrow r-1}=A_{2 \rightarrow r} \sigma_{r \leftarrow 1}^{-1}$ and inductive relations (7) for the projectors $A_{1 \rightarrow r}$ one can rewrite (20) in the form (16).

\section{Comments.}

i. For general $\sigma_{k l}^{i j}$ and $C_{j k}^{i}$ it is rather difficult to solve equations (16) explicitly. However for the case $\sigma^{2}=1$ the main equations (16) become simpler and the general solution for $Q$ can be found. Indeed the relation (16) for $r=2$ gives

$$
A_{1 \rightarrow 3} X_{\mid 123>}^{<12 \mid}\left(\mathbf{1}-\sigma_{12}\right)=A_{1 \rightarrow 3}\left(\sigma_{23} \sigma_{12}-\mathbf{1}\right) X_{\mid 23>}^{<2 \mid} .
$$

For $\sigma^{2}=1$ we have $A_{1 \rightarrow 3}\left(\sigma_{23} \sigma_{12}-\mathbf{1}\right)=0$ and therefore $Q_{(r)}=0$ for $r \geq 2$. Thus the BRST operator (14) has the familiar form

$$
Q=\Omega^{<1 \mid} \chi_{\mid 1>}-\Omega^{<2 \mid} \otimes \Omega^{<1 \mid} C_{\mid 12>}^{<1 \mid} \gamma_{\mid 1>} .
$$


In the case when the matrix $\sigma$ is the (super)-permutation matrix the algebra $\Gamma^{\wedge}$ with the differential (13) becomes the standard complex for the Lie (super)-algebra $\Gamma$ (see e.g. [7]).

In general, for $\sigma^{2} \neq 1$, the sum in (14) will be limited only by the height $h$ of the operator $\sigma$.

Below we present an explicit form for $Q$ for the standard quantum deformation $\Gamma=U_{q}(g l(N))$ of the universal enveloping algebra of the Lie algebra $g l(N)\left(\sigma^{2} \neq 1\right.$ in this case $)$.

ii. When the algebra (11) is a Hopf algebra, the algebraic structure (6), (8) (11) is related to the differential calculus on quantum groups (see [2], 8], [9]). The BRST operator $Q$ given by (14) generates the differential $d$ (introduced in [2]) on the algebra dual to $\Gamma^{\wedge}$.

6. Example. The BRST operator $Q$ for the quantum algebra $\Gamma=$ $U_{q}(g l(N))$.

The quantum algebra $U_{q}(g l(N))$ is defined (as a Hopf algebra) by the relations [5]

$$
\begin{gathered}
\hat{R} L_{2}^{ \pm} L_{1}^{ \pm}=L_{2}^{ \pm} L_{1}^{ \pm} \hat{R}, \quad \hat{R} L_{2}^{+} L_{1}^{-}=L_{2}^{-} L_{1}^{+} \hat{R} \\
\Delta\left(L^{ \pm}\right)=L^{ \pm} \otimes L^{ \pm}, \quad \varepsilon\left(L^{ \pm}\right)=\mathbf{1}, \quad S\left(L^{ \pm}\right)=\left(L^{ \pm}\right)^{-1},
\end{gathered}
$$

where elements of the $N \times N$ matrices $\left(L^{ \pm}\right)_{j}^{i}$ are generators of $U_{q}(g l(N))$; the matrices $L^{+}$and $L^{-}$are respectively upper and lower triangular, their diagonal elements are related by $\left(L^{+}\right)_{i}^{i}\left(L^{-}\right)_{i}^{i}=1$ for all $i$. The matrix $\hat{R}$ is defined as $\hat{R}:=\hat{R}_{12}=P_{12} R_{12}\left(P_{12}\right.$ is the permutation matrix); The matrix $R_{12}$ is the standard Drinfeld-Jimbo $R$-matrix for $G L_{q}(N)$,

$$
R_{12}=R_{j_{1}, j_{2}}^{i_{1}, i_{2}}=\delta_{j_{1}}^{i_{1}} \delta_{j_{2}}^{i_{2}}\left(1+(q-1) \delta^{i_{1} i_{2}}\right)+\left(q-q^{-1}\right) \delta_{j_{2}}^{i_{1}} \delta_{j_{1}}^{i_{2}} \Theta_{i_{1} i_{2}},
$$

where

$$
\Theta_{i j}= \begin{cases}1 & \text { if } i>j, \\ 0 & \text { if } i \leq j .\end{cases}
$$

This $R$-matrix satisfies the Hecke condition $\hat{R}^{2}=\lambda \hat{R}+\mathbf{1}$, where $\lambda=\left(q-q^{-1}\right)$ and $q$ is a parameter of deformation.

The generators of the algebra $\Gamma$ are defined by the formula [10], [11], [9]

$$
\chi_{k}^{l}=\frac{1}{\lambda}\left[\left(D^{-1}\right)_{k}^{l}-\left(D^{-1}\right)_{i}^{j} f_{k j}^{l i}\right]
$$


Here $f_{k j}^{l i}=L^{-i} S\left(L^{+l}{ }_{j}\right)$ and the numerical matrix $D$ can be found by means of relations

$$
\operatorname{Tr}_{2} \hat{R}_{12} \Psi_{23}=P_{13}=\operatorname{Tr}_{2} \Psi_{12} \hat{R}_{23}, \quad D_{1}:=\operatorname{Tr}_{2} \Psi_{12} \Rightarrow \operatorname{Tr}_{1}\left(D_{1}^{-1} \hat{R}^{-1}\right)=\mathbf{1}_{2},
$$

where $T r_{1}$ and $T r_{2}$ denote the traces over first and second spaces.

It is convenient to write down the complete set of commutation relations for the exterior algebra $\Gamma^{\wedge}[\Omega]$ in terms of generators

$$
\begin{gathered}
L_{j}^{i}=\left(L^{+}\right)_{k}^{i} S\left(\left(L^{-}\right)_{j}^{k}\right)=\delta_{j}^{i}-\lambda S^{-1}\left(\chi_{k}^{i}\right) D_{j}^{k}, \\
J_{n}^{i}=-S^{-1}\left(f_{j l}^{i k}\right) \gamma_{k}^{l} D_{n}^{j}, \quad \omega_{j}^{i}=\Omega_{m}^{k} f_{k j}^{m i} .
\end{gathered}
$$

The indices now are pairs of indices; the roles of the elements $\chi_{i}, \gamma_{j}$ and $\Omega^{k}$ are played by the generators $\chi_{j}^{i}, \gamma_{j}^{i}$ and $\Omega_{j}^{i}$ respectively.

The commutation relations are [12], [1], [9]:

$$
\begin{gathered}
\omega_{2} \hat{R}^{-1} \omega_{2} \hat{R}=-\hat{R}^{-1} \omega_{2} \hat{R}^{-1} \omega_{2}, \quad \omega_{2} \hat{R} L_{2} \hat{R}=\hat{R} L_{2} \hat{R} \omega_{2}, \\
\omega_{2} \hat{R} J_{2} \hat{R}+\hat{R} J_{2} \hat{R} \omega_{2}=-\hat{R}, \quad L_{2} \hat{R} L_{2} \hat{R}=\hat{R} L_{2} \hat{R} L_{2}, \\
J_{2} \hat{R} L_{2} \hat{R}=\hat{R} L_{2} \hat{R} J_{2}, \quad J_{2} \hat{R} J_{2} \hat{R}=-\hat{R}^{-1} J_{2} \hat{R} J_{2} .
\end{gathered}
$$

Now the construction of the BRST operator $Q$ is in order. To begin we find the first term in the sum (14):

$$
\Omega_{m}^{k} \chi_{k}^{m}=\frac{1}{\lambda} \operatorname{Tr}_{q}(\omega(L-\mathbf{1}))
$$

where we have introduced the quantum trace $\operatorname{Tr}_{q}(X):=\operatorname{Tr}\left(D^{-1} X\right)$. Then one can resolve the chain of the recurrent relations (16) where we have to substitute the expressions for the structure constants

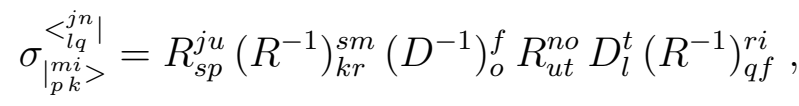

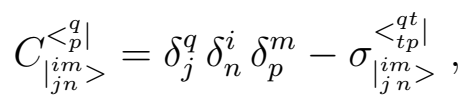

and find the set of coefficients $X_{\mid 1 \ldots r+1>}^{<1 \ldots r \mid}$. After straightforward but tiresome calculations one can obtain the following result:

$$
Q=\operatorname{Tr}_{q}\left(\omega(L-\mathbf{1}) / \lambda-\omega L(\omega J)+\lambda \omega L(\omega J)^{2}-\lambda^{2} \omega L(\omega J)^{3}+\ldots\right)
$$




$$
\begin{gathered}
=\operatorname{Tr}_{q}\left(\omega(L-\mathbf{1}) / \lambda-\omega L(\omega J)(\mathbf{1}+\lambda \omega J)^{-1}\right) \\
=-\frac{1}{\lambda} \operatorname{Tr}_{q}(\omega)+\frac{1}{\lambda} \operatorname{Tr}_{q}(W),
\end{gathered}
$$

where $W=\omega L(\mathbf{1}+\lambda \omega J)^{-1}$ and the sum in the first line of (28) is limited by the requirement that monomials of $\omega$ 's of the order $N^{2}+1$ are equal to zero.

One can check directly that the operator $Q$ given by (28) satisfies:

$$
Q^{2}=0, \quad[Q, L]=0, \quad[Q, J]_{+}=\frac{1}{\lambda}(\mathbf{1}-L) .
$$

To obtain these relations one has to use identities

$$
\operatorname{Tr}_{q}(X) \mathbf{1}_{2}=\operatorname{Tr}_{q 1}\left(\hat{R}^{ \pm 1} X_{2} \hat{R}^{\mp 1}\right)
$$

and relations

$$
\hat{R} W_{2} \hat{R}^{-1} \omega_{2}=-\omega_{2} \hat{R}^{-1} W_{2} \hat{R}
$$

$$
\begin{aligned}
& \hat{R} W_{2} \hat{R}^{-1} W_{2}=-W_{2} \hat{R}^{-1} W_{2} \hat{R}^{-1}, \quad \hat{R}^{-1} W_{2} \hat{R} L_{2}=L_{2} \hat{R} W_{2} \hat{R}^{-1}, \\
& J_{2} \hat{R} W_{2} \hat{R}^{-1}+\hat{R}^{-1} W_{2} \hat{R} J_{2}=-L_{2}(\mathbf{1}+\lambda \omega J)_{2}^{-1} \hat{R}^{-1}(\mathbf{1}+\lambda \omega J)_{2},
\end{aligned}
$$

which follow from (24)-(26).

Remark. The operator $Q$ given by (28) has the correct classical limit for $q \rightarrow 1(\lambda \rightarrow 0, L \rightarrow \mathbf{1}+\lambda \tilde{\chi}, \omega \rightarrow \tilde{\omega}, J \rightarrow \tilde{\gamma})$

$$
Q \rightarrow Q_{c l}=\operatorname{Tr}\left(\tilde{\omega} \tilde{\chi}+\tilde{\omega}^{2} \tilde{\gamma}\right)=\operatorname{Tr}(\tilde{\omega} X-\tilde{\omega} \tilde{\gamma} \tilde{\omega})
$$

where $X:=\tilde{\chi}+\tilde{\omega} \tilde{\gamma}+\tilde{\gamma} \tilde{\omega}$ and the classical algebra is

$$
\begin{gathered}
{\left[\tilde{\omega}_{2}, \tilde{\gamma}_{1}\right]_{+}=P_{12}, \quad\left[\tilde{\omega}_{2}, \tilde{\omega}_{1}\right]_{+}=0=\left[\tilde{\gamma}_{2}, \tilde{\gamma}_{1}\right]_{+}} \\
{\left[X_{2}, X_{1}\right]=P_{12}\left(X_{2}-X_{1}\right), \quad\left[X_{2}, \tilde{\omega}_{1}\right]=0=\left[X_{2}, \tilde{\gamma}_{1}\right] .}
\end{gathered}
$$




\section{Acknowledgements.}

We thank P. N. Pyatov, R. Stora and A. A. Vladimirov for valuable discussions. AI also thanks K. Schmudgen and his team for useful comments and hospitality at Leipzig University. This work was partially supported by the RFBR grant 98-01-2033, the CNRS grant PICS-608 and the VotrubaBlokhintsev program. The work of AI was also supported by the RFBR grant 00-01-00299.

\section{References}

[1] S. L. Woronowicz, Publ. RIMS Kyoto 23 (1987) 117.

[2] S. L. Woronowicz, Comm.Math.Phys.122 (1989) 125-170.

[3] D. Bernard, Phys. Lett. B 260 (1991) 389.

[4] P. Aschieri, L. Castellani, Int.J.Mod.Phys. A, 8 (1993) 1667.

[5] L. D. Faddeev, N. Yu. Reshetikhin, and L. A. Takhtajan, Algebra $i$ Analiz 1 No.1 178 (1989); English transl: Leningr.Math.J. 1193 (1990).

[6] D. I. Gurevich, Algebra i Analiz 2 No.4 (1990) 119.

[7] N. Jacobson, Lie Algebras, New York - London, Interscience, 1962.

[8] P. Schupp, preprint LMU-TPW 94-8 (1994), in: Como Quantum Groups (1994) pp. 507-524, hep-th/9408170

[9] O. V. Radko, A. A. Vladimirov, J.Math.Phys. 38 (1997) 5434.

[10] B. Jurco, Lett. Math. Phys. 22 (1991) p. 177.

[11] A. P. Isaev, "R-Matrix Approach to Differential Calculus on Quantum Groups", Fiz. Elem. Chastits At. Yadra 28 No. 3, (1997) 685-752, English translation in Phys. Part. Nucl. 28 (3) (1997) 267.

[12] P. Schupp, P. Watts and B. Zumino, Lett. Math. Phys. 25 (1992) 139. 